\title{
Experiencias de trabajo colaborativo mediante Tecnologías de la Información y la Comunicación entre profesores 1
}

\author{
Marcos C. Gonzálezi, Sonia C. Martínii \& Jorge M. Arribaiii \\ Universidad de Salamanca, España
}

\section{Resumen}

Durante los años 2011-2015, el Grupo de Investigación-Innovación en Tecnología Educativa de la Facultad de Educación de la Universidad de Salamanca ha llevado a cabo una investigación, financiada por el Ministerio de Ciencia e Innovación, orientada al conocimiento de las concepciones, metodologías y prácticas profesionales de los docentes de Educación Primaria y Educación Secundaria Obligatoria (ESO) sobre el aprendizaje colaborativo por medio de las Tecnologías de la Información y la Comunicación, en el contexto español, en la llamada Escuela 2.0. Este artículo se centra en el análisis de una parte de los resultados: el estudio de las experiencias de trabajo colaborativo mediado por TIC que se desarrollan entre los profesores de Primaria y ESO. Se ha utilizado un enfoque metodológico mixto (cuantitativo y cualitativo) y un método no experimental y descriptivo. Como principal conclusión destacamos que la cultura de trabajo colaborativo mediado por TIC entre docentes es muy pobre.

Palabras-clave

Innovación educativa; Escuela 2.0; Trabajo colaborativo; TIC

\section{Introducción}

Este artículo expone una parte de la investigación titulada "Aprendizaje colaborativo a través de las Tecnologías de la Información y la Comunicación 
en el contexto de la Escuela 2.0", llevada a cabo por el Grupo de Investigación-Innovación en Tecnología Educativa de la Facultad de Educación de la Universidad de Salamanca (GITE-USAL), durante los años 2011 al 2015.

En una primera fase del proyecto se trató de conocer las concepciones que los profesores de tercer ciclo de Primaria y primer ciclo de Educación Secundaria Obligatoria tenían sobre la metodología del aprendizaje colaborativo, las experiencias de trabajo colaborativo mediante las TIC que desarrollaban en el aula y las actividades de trabajo colaborativo que realizaba este profesorado con otros compañeros, bien de su propio centro o de otros, buscando su desarrollo profesional y la formación permanente.

En un segundo momento, por medio del estudio de casos, se buscó profundizar en las características de los procesos que se generan en distintos tipos de metodologías colaborativas, especialmente a través de la utilización de redes de comunicación asíncrona escrita, así como en los resultados de aprendizaje (competencias) que se obtienen y desarrollan en los alumnos.

\section{Fundamentación teórica}

La escuela sociocultural, dentro de las perspectivas teóricas del modelo pedagógico constructivista, sostiene que a la hora de aprender uno de los elementos más importantes es la socialización de experiencias y conocimientos. Por ello, una de las mejores formas de aprender es hacerlo junto con otros (Berenice, De Aguinaga, \& Ávila, 2010).

La denominada Web 2.0, concepto que pasó a caracterizar la Internet no solamente como un espacio de lectura, sino también de escritura, y que enfatiza un nuevo modelo comunicativo en el que priman los procesos de interrelación entre personas o grupos, a través del empleo de una gran cantidad y variedad de herramientas para gestionar contenidos e información, constituye un buen aliado para construir conocimientos de forma colaborativa y puede favorecer o impulsar esta forma de aprender con los demás (Cabezas \& Casillas, 2009; Cebrián, 2008; De la Torre, 2006). Según Marquès (2007), las aplicaciones que ofrece este tipo de Web pueden ser un instrumento idóneo para favorecer metodologías socio-constructivistas centradas en los profesores y en el aprendizaje autónomo y colaborativo. 
Esta Web, también denominada Web social, se basa en comunidades de usuarios con intereses comunes que utilizan una serie de servicios como redes sociales, blogs, wikis, podcasts, etc., fomentándose una colaboración y un intercambio ágil y eficaz de la información entre los usuarios, creando éstos contenidos de forma colaborativa.

Con el término Web 2.0, subrayamos un cambio de paradigma sobre la concepción de Internet y sus funcionalidades, que ahora abandonan su marcada unidireccionalidad y se orientan más a facilitar la máxima interacción entre los usuarios y el desarrollo de redes sociales (tecnologías sociales) donde puedan expresarse y opinar, buscar y recibir información de interés, colaborar y crear conocimiento (conocimiento social), compartir contenidos. (Marquès, 2007)

Este componente de socialización es la gran aportación de la Web 2.0, donde la suma de esfuerzos individuales da lugar a una red de conocimiento compartido, y a mayor número de personas compartiendo, mayor utilidad de los servicios. Para Castells (2011), en las últimas dos décadas se ha producido una revolución tecnológica ejemplificada por Internet y las redes móviles, donde el sistema de mensajes es múltiple, de muchos a muchos. Esto hace posible que la comunicación entre docentes sea más fácil y fluida.

El término 'trabajo colaborativo' surge en la década de los ochenta para referirse a un entorno de trabajo donde los participantes organizados en grupo colaboran y se ayudan para conseguir un objetivo, apoyados con el uso de las Tecnologías de la Información y la Comunicación (Rosario, 2008). Johnson \& Johnson (1987) ofrecen una definición en la cual se pone el énfasis de la interdependencia que hay entre el esfuerzo y aprendizaje individual y el grupal, ya que cada miembro del grupo es responsable tanto de su aprendizaje como del de los restantes miembros. Se puede considerar que en el trabajo colaborativo se produce una unión e intercambio de esfuerzos entre los integrantes que conforman un grupo (en este caso entre profesores), de tal manera que el objetivo común y grupal que se persigue produzca, al final del proceso, un beneficio individual en todos y cada uno de los participantes.

Uno de los objetivos fundamentales que se persiguen a la hora de utilizar esta forma de trabajo entre profesores es el de promover una adecuada formación y un apropiado desempeño laboral a partir del intercambio de ideas y acciones entre los docentes implicados (Jiménez, 2009). La colaboración contribuye a gestionar el conocimiento de una manera 
eficaz, ayuda al equilibrio de los componentes y permite el trabajo activo y comprometido, otorgando voz y participación a todos por igual (Trujillo, Cáceres, Hinojo, \& Aznar, 2011). El trabajo colaborativo permite el logro de objetivos que son cualitativamente más ricos en contenidos y asegura la calidad y la exactitud en las ideas y soluciones planteadas, además de propiciar la generación de conocimiento, debido a que el profesor se ve involucrado en el desarrollo de investigaciones en donde su aportación es muy valiosa al no permanecer como un sujeto pasivo que solo capta información (Rosario, 2008).

Para que el trabajo colaborativo entre profesores tenga éxito es necesario que los docentes; se ayuden mutuamente y tengan claro que solo se conseguirán los objetivos individuales si se consiguen los grupales (interdependencia positiva); se apoyen activamente y compartan recursos (interacción promotora); asuman su responsabilidad individual y grupal; aprendan competencias académicas, interpersonales, y de trabajo grupal; y evalúen la efectividad del grupo en la consecución de los objetivos propuestos (Johnson, Johnson, \& Smith, 1998).

Que los docentes trabajen de forma colaborativa supone que estos puedan aprovecharse de una serie de beneficios, tales como: promover las relaciones entre los participantes; aumentar la motivación y la autoestima; desarrollar habilidades interpersonales y estrategias para resolver conflictos; promover el respeto, la tolerancia, la flexibilidad y la apertura hacia los demás; enseñar a compartir responsabilidades; enseñar a organizarse y a dividir las tareas y los roles para lograr un mejor resultado; facilitar la corrección al dar cabida a la confrontación del trabajo individual con lo que hacen los demás miembros del grupo; brindar el espacio para superar las dificultades que alguien pueda tener en un ambiente de compañerismo y confianza (Brito, 2004).

En algunas investigaciones sobre la cultura colaborativa entre docentes (Arbaugh, 2003; Arnaiz, Herrero, Garrido, \& De Haro, 1999; Austin, 2001; Muñoz, Quintero, \& Munévar, 2002; Williams, Prestage, \& Bedward, 2001) se pone de manifiesto que: la colaboración ha sido una metodología utilizada más en la inclusión de alumnos que como un método de trabajo en el aula "normal"; el trabajo colaborativo entre docentes está más desarrollado en la teoría que en la práctica; el tiempo, el sentimiento de amenaza y temor, 
los valores y las costumbres son los principales obstáculos para la relación profesional entre docentes; la colaboración es una forma de enriquecimiento mutuo que permite desarrollar habilidades docentes.

En esta investigación que presentamos, nos centramos en la colaboración mediada por TIC entre profesores como un medio de desarrollo profesional y de formación permanente.

\section{Metodología}

La metodología empleada en esta parte de la investigación se caracteriza, en general, por estar comprendida dentro de un paradigma positivista, ya que trata de diagnosticar el objeto de estudio, además de utilizar técnicas cuantitativas y cualitativas a la hora de realizar el tratamiento estadístico de los datos. Por otro lado, se utiliza un método no experimental y descriptivo, empleando para la recogida de la información la técnica del cuestionario.

\subsection{Objetivo}

El objetivo de la investigación que se pretende conseguir y que concierne a los datos expuestos es el de analizar las prácticas profesionales de trabajo colaborativo que realizan los docentes con otros profesores utilizando TIC, buscando su desarrollo profesional y la formación permanente.

\subsection{Muestra}

La población queda definida por los profesores de los centros de Primaria que imparten asignaturas en el tercer ciclo y los profesores de los centros de Secundaria que imparten clase en $1^{\circ}$ y $2^{\circ}$ de la ESO, en la comunidad autónoma de Castilla y León. El total de estos centros fue 49 con una certificación TIC de nivel 5 y 149 centros con una certificación TIC de nivel 4 (niveles superiores de certificación de incorporación de las TIC al centro educativo). Esta certificación para centros educativos en aplicación de Tecnologías de la Información y la Comunicación, que es otorgada por la Consejería de Educación de Castilla y León, tiene como objeto el reconocimiento de aquellos centros educativos que se muestren como 
referente destacado en la integración, aplicación, fomento, desarrollo e innovación de las TIC en el marco del desarrollo de su actividad escolar. Dicha certificación se basa principalmente en promover la aplicación de las TIC en las áreas de integración curricular, infraestructuras y equipamiento, ámbito formativo, gestión y administración, y comunicaciones y acción social. Para ser otorgada hay que cumplir los siguientes criterios:

- Nivel 4 (Valor global entre 3 y 4) - Bueno: La aplicación de TIC es generalizada. Existen equipamientos e infraestructuras en el $80 \%$ de los procesos. Existen planes de integración puestos en marcha tanto en la actualidad como en el futuro. La formación en TIC es un proceso integrado y clave. La implicación es alta, así como el reconocimiento de las ventajas y el valor añadido de la aplicación de las TIC.

- Nivel 5 (Valor global entre 4 y 5) - Excelente: Existe una integración excelente de las TIC en el centro. Los equipamientos cumplen con fases de renovación y están integrados en el 100\% de los procesos implicados. Los planes asumen las herramientas como un proceso integrado, logrando mejoras continuas en los resultados. Se proporciona formación continua y asociada al uso y entorno TIC (aplicación de herramientas en formación). La implicación y el conocimiento en TIC es muy alto y se aplican en la totalidad de los procesos (ORDEN EDU/1761/2009).

La selección de la muestra se realizó por medio de un muestreo no probabilístico, siguiendo un proceso que constó de dos fases. Por un lado se contactó telefónicamente con los directores de los 149 centros de nivel 4, pidiéndoles que pasaran la información relativa a la investigación al profesorado, facilitándoles además la dirección Web donde podrían contestar al cuestionario online. Por otro lado, y en cuanto a los centros de nivel 5, también se contactó telefónicamente con los directores, procediendo de igual manera que en los otros centros. No obstante, hubo una parte de los mismos que nos permitieron visitas presenciales (para llevar a cabo la segunda fase de la investigación, descrita en la introducción), pudiendo solicitar de nuevo al profesorado que respondiera al cuestionario.

La muestra final la han constituido un total de 185 profesores, de los cuales el 76\% imparte docencia en Educación Primaria y el 50\% en Educación 
Secundaria Obligatoria (habiendo profesores que imparten docencia en ambas etapas). Un $65 \%$ han sido profesoras, y un $35 \%$ profesores. Como datos identificativos de la muestra, también reseñar que el 55\% de los docentes desarrolla su trabajo en centros rurales y, en su mayoría, posee una dilatada experiencia profesional, tal como se muestra en el siguiente gráfico.

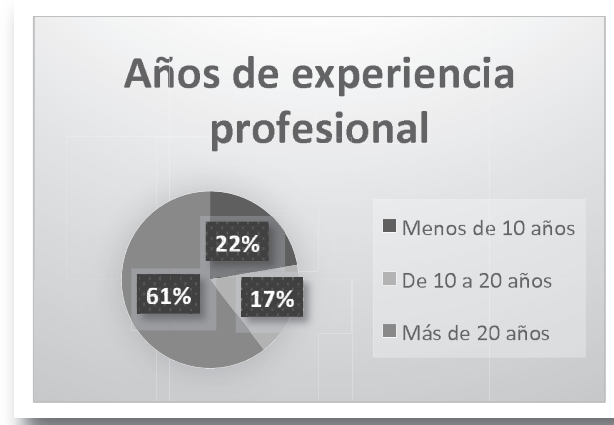

\section{Gráfico 1 - Distribución del profesorado atendiendo a sus años de experiencia profesional}

\subsection{Instrumento}

Los datos que analizamos en este artículo fueron obtenidos por medio de un cuestionario utilizado en la investigación y creado con la herramienta formulario del servicio Google Drive. Se trata de una herramienta colaborativa que permite almacenar datos con un nivel alto de seguridad, y que nos sirvió para crear un cuestionario de fácil distribución y acceso, que podía ser contestado desde cualquier dispositivo y desde cualquier lugar. Dicho cuestionario consta de cuatro partes:

- La primera incluye una serie de datos de identificación como el sexo, el cargo en el centro (profesor/a, director), el nivel educativo en el que se imparte docencia (Primaria y Secundaria), los años de experiencia profesional, la situación del centro (provincias de la Comunidad de Castilla y León), el tipo (centro rural agrupado, centro de Educación Infantil y Primaria, Instituto de Educación Secundaria, Instituto de Educación Secundaria Obligatoria), el 
ámbito rural o urbano, la acreditación TIC del centro (nivel cuatro bueno; nivel cinco - excelente) y la media de alumnos en clase.

- La segunda parte está dirigida a conocer las opiniones del profesorado sobre el aprendizaje colaborativo, independientemente de que lo hubiesen llevado a la práctica o no, recopilando también información acerca de sus conocimientos sobre herramientas online que facilitan el trabajo colaborativo.

- La tercera se dirige a aquellos profesores que realizan experiencias de trabajo colaborativo a través de las TIC con sus estudiantes, incidiendo en cuestiones relacionadas con el tipo de experiencias o actividades, las herramientas utilizadas y los criterios organizativos considerados.

- La cuarta y última parte es dirigida a aquellos profesores que realizan experiencias de trabajo colaborativo con sus colegas.

En este artículo nos vamos a centrar en analizar la cuarta parte del cuestionario online. Ésta consta de 6 ítems, de los cuales 1 es de corte cuantitativo y el resto son preguntas abiertas para analizar cualitativamente. En concreto, se pregunta a los profesores sobre la frecuencia con la que realizan trabajo colaborativo mediado por TIC con otros compañeros, sobre qué experiencias de trabajo colaborativo han realizado con sus colegas a través de TIC y cuál es su valoración, sobre qué plataformas han utilizado y por qué, así como sobre los beneficios e inconvenientes que han encontrado al llevar a cabo este tipo de experiencias.

\subsection{Procedimiento}

El análisis de los datos obtenidos, y que presentamos en este artículo, ha requerido el empleo de metodologías cuantitativa y cualitativa, lo que permite un enriquecimiento de la información obtenida.

Los datos de carácter cuantitativo se han analizado, desde perspectivas descriptivas e inferenciales, con la aplicación informática SPSS. La información cualitativa ha sido procesada y analizada utilizando el programa informático ATLAS.ti. Para ello, se han introducido en el programa 6 preguntas (correspondientes a 5 ítems), susceptibles de ser tratadas cualitativamente. Estas preguntas y sus respectivas respuestas conforman los 
documentos primarios. De ellos, se han ido seleccionando partes de las respuestas, llamadas citas, que se han ido clasificando y agrupando bajo códigos comunes. De esta reducción de datos se obtienen una serie de códigos que se han agrupado en familias y se han establecido entre ellos relaciones de distinta naturaleza.

Si bien el análisis cualitativo requiere de un gran trabajo previo en cuanto a preparación de los documentos y de categorización y codificación, la utilización de estos programas permite dotar de un rigor científico al análisis cualitativo, equiparándolo al cuantitativo. En nuestro caso, la utilización de este programa nos ha permitido tratar de una manera más sistemática y profunda los datos de corte cualitativo que obtuvimos.

En el siguiente apartado de resultados, se puede apreciar claramente todo el proceso descrito.

\section{Resultados}

Es necesario aclarar una serie de cuestiones sobre el número de respuestas dadas al apartado cuarto del cuestionario. Tal y como se ha indicado con anterioridad, la muestra fue de 185 profesores. Pero para poder contestar a los bloques de preguntas de la tercera y cuarta parte se planteó una pregunta filtro: ¿ha realizado experiencias de trabajo colaborativo a través de TIC con otros profesores? Dependiendo de si los profesores respondían sí o no a estas cuestión, pasaban o no a responder a las cuestiones planteadas en estas dos últimas partes. De este modo, de los 185 profesores fueron 60 los que pasaron a responder las cuestiones sobre si habían realizado experiencias de trabajo colaborativo a través de TIC con colegas. Además, las preguntas de este bloque no eran obligatorias, por lo que el número de respuestas, a veces, se ve todavía más mermado.

Son pocos los profesores $(32,43 \%)$ que han trabajado colaborativamente con otros compañeros utilizando para ello las TIC. Podemos decir que la cultura del trabajo colaborativo utilizando TIC es baja cuando se trata de trabajar colaborativamente con otros colegas. 


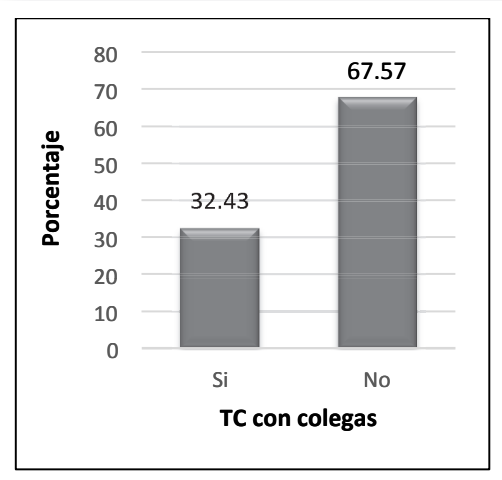

\section{Gráfico 2 - Profesores que han realizado trabajo colaborativo a través de TIC con otros profesores}

Tratando de profundizar en estos datos y relacionándolos con los de las otras partes del cuestionario, en la siguiente tabla se muestran los profesores que sí han realizado experiencias de trabajo colaborativo a través de TIC con estudiantes y profesores, los que las han realizado con estudiantes pero no con compañeros, los que han realizado experiencias de trabajo colaborativo a través de TIC con colegas pero no con estudiantes, y los que no han realizado este tipo de experiencias ni con estudiantes ni con otros profesores.

Tabla 1 - ¿Han realizado experiencias de TC mediado por TIC?

\begin{tabular}{|c|c|c|c|}
\hline \multicolumn{2}{|c|}{$\begin{array}{l}\text { ¿Ha realizado experiencias de } \\
\text { trabajo colaborativo a través de } \\
\text { TIC con sus estudiantes? }\end{array}$} & \multicolumn{2}{|c|}{$\begin{array}{l}\text { ¿Ha realizado experiencias de } \\
\text { trabajo colaborativo a través de } \\
\text { TIC con colegas? }\end{array}$} \\
\hline \multirow{2}{*}{ Si } & \multirow{2}{*}{$98(52,97 \%)$} & $\mathrm{Si}$ & $46(24,86 \%)$ \\
\hline & & No & $52(28,11 \%)$ \\
\hline \multirow{2}{*}{ No } & \multirow{2}{*}{$87(47,03 \%)$} & $\mathrm{Si}$ & $14(7,57 \%)$ \\
\hline & & No & $73(39,46 \%)$ \\
\hline
\end{tabular}

Un porcentaje elevado de profesores, casi el $40 \%$, no han realizado experiencias de trabajo colaborativo a través de TIC ni con estudiantes ni con 
profesores, lo que refuerza la idea anteriormente señalada acerca de la escasa presencia de esta metodología de trabajo. Como dato positivo podemos señalar que casi un $25 \%$ realiza este tipo de experiencias tanto con estudiantes como con colegas. Es curioso también el porcentaje importante de profesores $(28,11 \%)$ que aun llevando a cabo esta metodología de trabajo con sus alumnos no la practica con sus compañeros. Por otro lado, son pocos los profesores que colaboran con sus colegas pero no realizan experiencia de trabajo colaborativo a través de las TIC con sus estudiantes $(7,57 \%)$.

A continuación se exponen los resultados obtenidos a partir de los análisis de los datos realizados y se muestran las opiniones de los profesores, siguiendo el orden de los ítems que aparecen en el instrumento de recogida de información.

\subsection{Frecuencia con la que realiza trabajo colaborativo mediado por TIC con otros profesores}

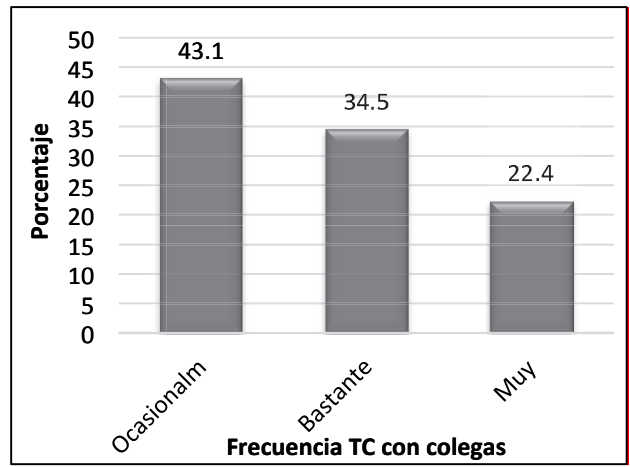

\section{Gráfico 3. Frecuencia con la que realiza TC mediado por TIC con otros} profesores

En cuanto a la frecuencia de este tipo de trabajo, la mayoría $(43,1 \%)$ lo realiza ocasionalmente, el $34,5 \%$ con bastante frecuencia y el $22,4 \%$ de manera muy frecuente. 


\subsection{Tipo de experiencias de trabajo colaborativo a través de TIC desarrolladas con otros profesores}

Esta pregunta fue respondida por un alto número de sujetos (57 de 60). En los siguientes gráficos (4 al 9), se muestran las respuestas organizadas por etiquetas. Los números que aparecen entre paréntesis en cada una de estas etiquetas se refieren, el primero de ellos, a la cantidad de citas a las que se aplica el código, y el segundo es un dígito que representa el orden que el programa estadístico ATLAS.ti asigna a los códigos y a las familias.

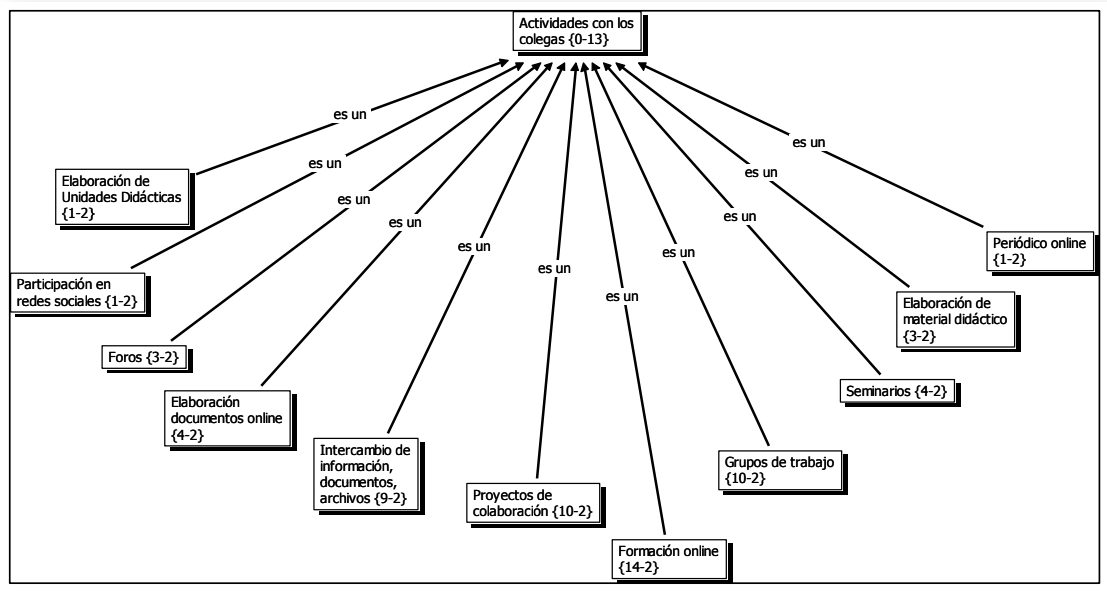

Gráfico 4. Experiencias de trabajo colaborativo a través de TIC con otros profesores

Las actividades o experiencias más desarrolladas por los profesores con sus homólogos son cursos de formación online. Destacan también los grupos de trabajo para llevar a cabo determinadas actividades y proyectos de colaboración, no sólo en el centro sino con otros centros, instituciones, o países. Por un lado, los docentes utilizan las TIC para formarse y mantener el contacto con otros profesores, para compartir experiencias y materiales (seminarios, grupos de trabajo, intercambio información, foros, etc.); y, por otro, utilizan las TIC para desarrollar proyectos didácticos con otros centros, aprovechando para ello las funcionalidades que ofrecen estas herramientas y que de otra manera serían casi imposibles de desarrollar. 
3.3. Valoración de las experiencias de trabajo colaborativo mediado por TIC desarrolladas con otros profesores

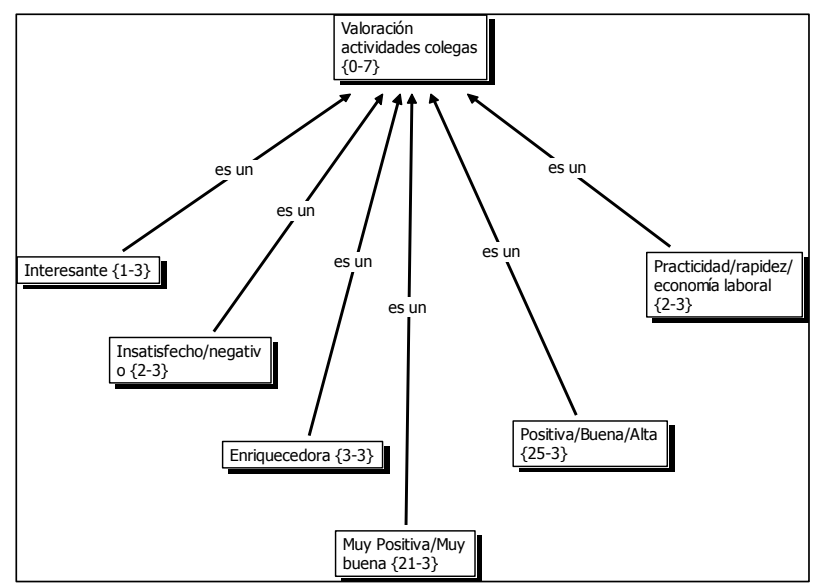

Gráfico 5 - Valoración de las experiencias colaborativas a través de TIC con otros profesores

En cuanto a la valoración que de estas actividades hace el profesorado, el número de respuestas también fue alto (55 de 60). Vemos como la valoración es mayoritariamente alta (Muy positiva/Muy buena, Positiva/Buena/Alta), quizás porque los que llevan a cabo estas prácticas saben de antemano los posibles beneficios que les pueden aportar.

\subsection{Plataformas utilizadas para el trabajo colaborativo a través de} TIC con otros docentes y motivos de su uso

En este ítem el número de respuestas es significativamente más bajo que en el resto de preguntas de este bloque (25 de 60), quizás porque los profesores ya mencionaron alguna de las herramientas TIC que utilizaban cuando nos hablaron de las actividades que realizaban con sus colegas a través de estas. 


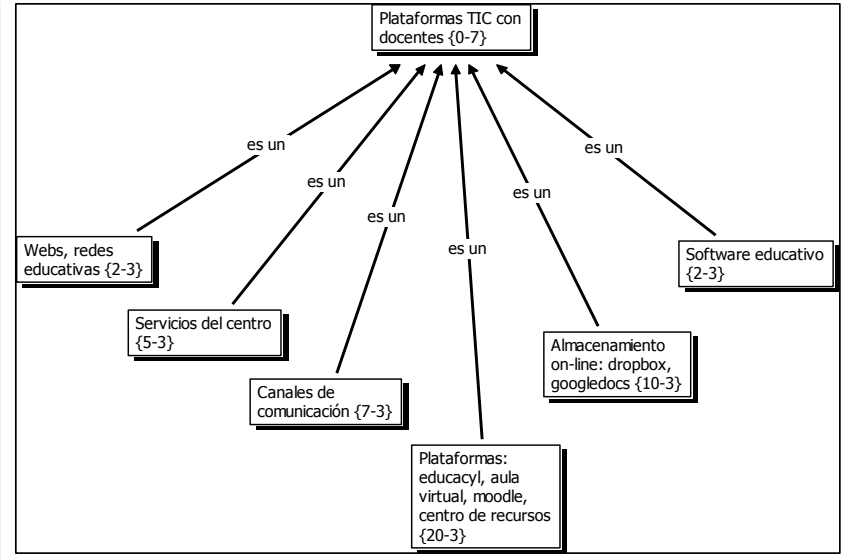

\section{Gráfico 6 - Plataformas TIC utilizadas en el trabajo colaborativo con otros docentes}

Muchos profesores mencionan como herramientas más utilizadas las plataformas o aulas virtuales, ya sean del centro, de la Consejería o de los centros de formación. Estas herramientas, más tradicionales, se complementan con otras propias de la Web 2.0 como son el almacenamiento online (Dropbox, Google Drive, One Drive, etc.) y canales de comunicación como los blogs y las redes sociales. Volvemos a observar que las experiencias y herramientas institucionales, o aquellas más estructuradas, son las más utilizadas por el profesorado, debido quizás por un lado a la necesidad u obligación de hacerlo, y por otro a que la formación que nace de la iniciativa propia no está muy extendida o no hay tiempo para llevarla a cabo.

En cuanto a los motivos que los profesores nos dieron para usar estas plataformas, todos destacan que éstas les hacen más fácil y cómoda la comunicación y el compartir recursos con sus compañeros. De este modo hemos establecido una relación de causalidad de estos motivos con los beneficios que aportan las TIC al trabajo colaborativo con colegas, los cuales se tratarán en el siguiente ítem. Es decir, viendo los motivos dados por los docentes y que estos tienen un carácter positivo, pensamos que estos contribuyen a los beneficios que supuestamente dicen los profesores aportan 
las TIC al trabajo colaborativo con los compañeros. Además, motivos y beneficios en más de una ocasión son los mismos, pudiendo, quizás, haber confundido los términos o el sentido de los mismos en dichas preguntas.

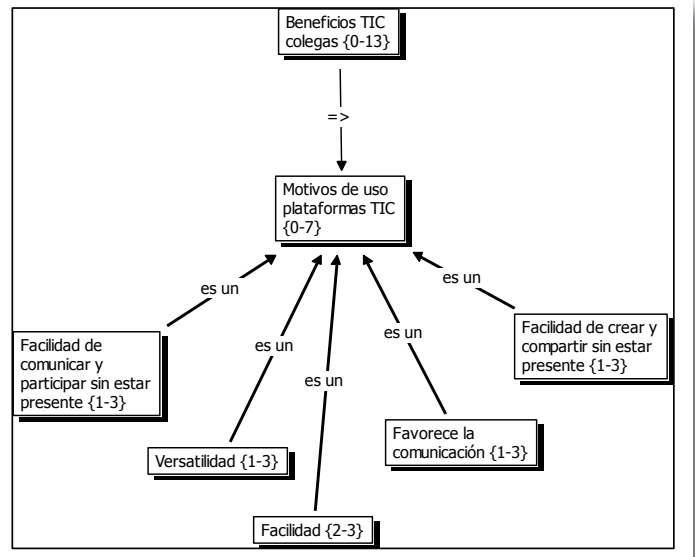

Gráfico 7 - Motivos de uso de las plataformas TIC utilizadas en el trabajo colaborativo con otros docentes

\subsection{Beneficios de las TIC para el trabajo colaborativo con otros docentes}

En este ítem de nuevo aumenta el número de repuestas (49 de 60), algo que veremos también en la siguiente cuestión sobre inconvenientes de las TIC en el trabajo colaborativo con colegas. Este tipo de preguntas suele ser utilizado, sobre todo en el caso de los profesionales de la educación, y más aún en el del uso de las TIC, para expresar no sólo los beneficios de estas, que se presuponen numerosos, sino para desahogarse de los problemas que les acarrean su utilización, que por otro lado también suelen ser numerosos y muy decisivos para el profesorado a la hora de adoptar su empleo o no en su práctica educativa diaria. 


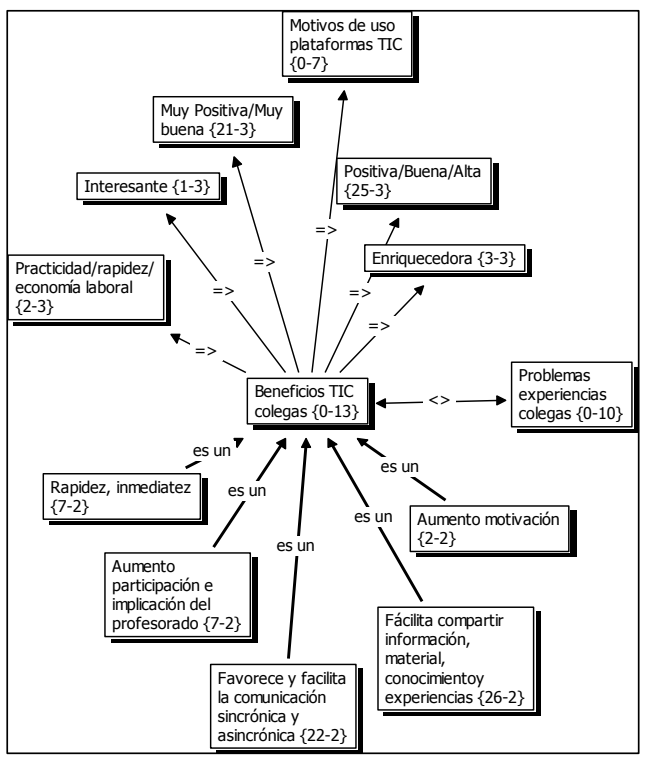

\section{Gráfico 8 - Beneficios de las TIC para el trabajo colaborativo con otros docentes}

En este gráfico aparece la relación de causalidad motivos-beneficios que hemos señalado con anterioridad. De este modo vuelven a aparecer como beneficios más comentados la facilidad y comodidad en la comunicación y en compartir recursos con sus compañeros, así como la rapidez y la inmediatez de este intercambio y comunicación. Destaca también cómo el profesorado señala que la implicación y motivación por este tipo de actividades aumenta, debido quizás a las facilidades comentadas.

3.6. Inconvenientes o problemas de las experiencias de trabajo colaborativo a través de las TIC con otros docentes

Como señalábamos en el anterior ítem, la participación de los profesores en esta cuestión vuelve a aumentar (48 de 98). 


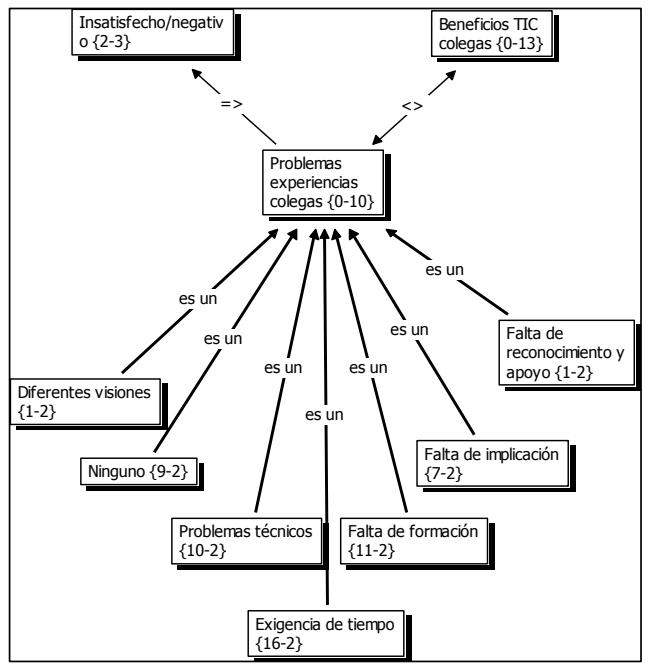

\section{Gráfico 9 - Inconvenientes en las experiencias del trabajo colaborativo con otros docentes a través de las TIC}

El principal problema que surge al usar las TIC en este tipo de experiencias es la exigencia de tiempo extra al margen casi siempre de su horario laboral. Este inconveniente es uno de los más señalados por los docentes a la hora valorar los problemas que acarrea el uso de las TIC. Para ellos, el emplear estas herramientas es un extra más a las tareas que les tienen encomendadas, y les supone una gran inversión de tiempo en preparar y llevar a cabo estas experiencias y, sobre todo, en formarse para dominar estas herramientas.

El segundo de los problemas más señalados es el de la falta de formación o conocimiento en el manejo de las herramientas que se utilizan. Este hecho provoca, según señalan los profesores, frustraciones, limitaciones, desigualdades, etc., entre los grupos de profesores que realizan experiencias colaborativas. Es también otro de los puntos que hacen que los profesores abandonen el uso de las TIC, al ver que les exigen no sólo mucho tiempo sino también una formación extra, inicial y permanente, algo a lo que quizás no están acostumbrados. 
Otro de los inconvenientes con los que los profesores se encuentran es con los problemas técnicos que surgen al manejar las TIC, tales como la calidad de conexión, funcionamiento de determinados programas, etc. Estas dificultades, sobre todo aquellas que no son subsanables por los propios docentes, suelen ralentizar este tipo de experiencias y además hacen que éstos sean más reacios a utilizarlas.

\section{Conclusiones y discusión}

La cultura del trabajo colaborativo mediada por TIC es escasa cuando se trata de trabajar colaborativamente con otros colegas. Así lo ponen de manifiesto los profesores de Castilla y León que han participado en esta investigación. Son pocos los que han trabajado colaborativamente con otros compañeros utilizando para ello las TIC. Un número elevado de profesores manifiestan que no han realizado este tipo de experiencias ni con estudiantes ni con profesores, lo que refuerza la idea anteriormente señalada acerca de la escasa presencia de esta forma de trabajar, enseñar y aprender. También destaca el número importante de profesores que, aun llevando a cabo este tipo de trabajo con sus alumnos, no lo practica con sus compañeros. Quizá esto pueda deberse a que, a pesar de los diversos intentos formativos en la última década para que los profesores utilicen las TIC en los centros escolares, éstos aún no tienen la suficiente confianza para utilizar estas tecnologías en su práctica profesional diaria (Sigalés, Mominó, Meneses, \& Badía, 2008).

Las actividades o experiencias más desarrolladas por los profesores con sus compañeros son cursos de formación online. Destacan también los grupos de trabajo para llevar a cabo determinadas actividades y proyectos de colaboración, no sólo en el centro sino con otros centros, instituciones, o países. Podemos decir que, fundamentalmente, los docentes utilizan las TIC para formarse y mantener el contacto con otros profesores, y para compartir experiencias y materiales entre ellos.

Muchos profesores mencionan como herramientas más utilizadas las plataformas o aulas virtuales, ya sean del centro, de la Consejería o de los centros de formación. Estas herramientas más tradicionales se complementan con otras propias de la Web 2.0 como son el almacenamiento online 
(Dropbox, Google Drive, Box, One Drive, etc.) y canales de comunicación como los blogs y las redes sociales. Volvemos a ver que las experiencias y herramientas institucionales o aquellas más estructuradas son las más utilizadas por el profesorado. Usar estas plataformas les hace más fácil comunicarse y compartir recursos con sus compañeros.

En cuanto a los beneficios derivados del uso de la TIC, los más comentados por el profesorado son la facilidad y comodidad en la comunicación y el poder compartir recursos con sus compañeros, así como la rapidez y la inmediatez de este intercambio y comunicación. Destacamos también cómo el profesorado señala que la implicación y motivación por este tipo de actividades aumenta.

El principal problema que surge al usar las TIC en este tipo de experiencias es la exigencia de tiempo extra al margen, casi siempre, de su horario laboral. Este inconveniente, uno de los más señalados por los docentes a la hora de valorar los problemas que acarrea el uso de este tipo de metodología mediada por TIC, no es novedoso porque podemos encontrar estudios que ya lo ponen de manifiesto hace muchos años (Serafí, 1999). Para ellos, usar estas herramientas es un añadido a las tareas que les tienen encomendadas y les supone una gran inversión de tiempo el hecho de tener que formarse para utilizarlas así como trabajar de forma colaborativa. Esto pone de manifiesto que los profesores aún no poseen las competencias necesarias respecto a las TIC (Ertmer \& Ottenbreif-Leftwich, 2010; Valverde, Garrido, \& Fernández, 2010), lo que constituye una barrera fundamental a la hora de integrarlas en su trabajo diario (Balanskat, Blamire, \& Kefala, 2006; Hew \& Brush, 2007). El segundo de los inconvenientes más señalados, y no sólo en esta investigación sino en otras realizadas recientemente con objetivos similares (Almerich, Suárez-Rodriguéz, Belloch, \& Bo, 2011; Almerich, Suárez-Rodriguéz, Jornet, \& Orellana, 2011; Álvarez-Rojo et al., 2011), es el de la falta de formación o conocimiento en el manejo de las herramientas que se utilizan. Este hecho provoca frustraciones, limitaciones, desigualdades, etc., entre los grupos de profesores que realizan experiencias colaborativas. Otro de los problemas con los que se encuentran es con los inconvenientes técnicos que surgen al manejar las TIC, tales como la calidad de conexión, funcionamiento de determinados programas, etc. 
En definitiva, al igual que otros autores (e.g. Montero Mesa, 2011), creemos que es necesario promover el trabajo colaborativo como una oportunidad formativa, no sólo entre los alumnos, sino también entre los profesores, profesionales que no están demasiado acostumbrados a trabajar de forma colaborativa, ni a compartir sus preocupaciones, y mucho menos utilizando para ello las TIC.

Las evidencias encontradas en esta investigación ponen de manifiesto que la cultura colaborativa docente es pobre cuando se trata de trabajar con los alumnos y muy pobre cuando se trata de trabajar con otros colegas, lo cual implica que, de alguna manera, la escuela como institución que ha de preparar a los futuros ciudadanos que han de incorporarse a la sociedad aún no está preparada para dar respuesta a la sociedad de la información y el conocimiento. En esta sociedad, donde la inteligencia colectiva y el conocimiento social son dos de sus pilares fundamentales, los docentes no pueden permanecer ajenos a ella. Es necesario romper con el aislamiento y el individualismo profesional del docente y apostar por intercambiar y compartir ideas, conocimientos y recursos. De la misma manera, es preciso potenciar el trabajo colaborativo entre los profesores como un medio que permita identificar problemas, discutirlos, reflexionar conjuntamente, experimentar alternativas de solución y evaluar las mismas. Y, para ello, las TIC tienen un gran potencial que puede facilitar enormemente el trabajo colaborativo entre los docentes.

En la sociedad de la información, el conocimiento y la comunicación, es necesario el trabajo colaborativo entre profesores de modo que estos participen en proyectos de innovación formando redes de docentes innovadores, compañeros del propio o de otros centros, que colaboran para llevar a cabo propuestas educativas novedosas en cuanto a la organización de las materias, coordinación de las mismas, puesta en práctica de nuevas metodologías didácticas, entre otras (García-Valcárcel, Hernández, \& Recamán, 2012).

\section{Nota}

1 Nota dos editores: este texto constitui uma versão corrigida do artigo originalmente publicado. Na página 80 , a expressão "muestreo probabilístico" (na versão original), foi substituída por "muestreo no probabilístico". 


\section{Referencias}

Almerich, G., Suárez-Rodriguéz, J. M., Jornet, J. M., \& Orellana, N. (2011). Las competencias y el uso de las Tecnologías de la Información y Comunicación (TIC) por el profesorado: Estructura dimensional. Revista Electrónica de Investigación Educativa, 13(1). Recuperado de:

http://redie.uabc.mx/redie/article/view/269

Almerich, G., Suárez-Rodriguéz, J. M., Belloch, C., \& Bo, R. M. (2011). Las necesidades formativas del profesorado en TIC: Perfiles formativos y elementos de complejidad. RELIEVE, 17(2). Recuperado de: http://www.uv.es/RELIEVE/ v17n2/RELIEVEv17n2_1.htm

Álvarez-Rojo, V., Romero, S., Gil-Flores, J., Rodríguez-Santero, J., Clares, J., Asensio, I. ...Salmeron-Vilchez, P. (2011). Necesidades de formación del profesorado universitario para la adaptación de su docencia al Espacio Europeo de Educación Superior (EEES). RELIEVE, 17(1). Recuperado de: http://www.uv.es/ RELIEVE/v17n1/RELIEVEv17n1_1.htm

Arnaiz, P., Herrero A. J., Garrido C., \& De Haro, R. (1999). Trabajo colaborativo entre profesores y atención a la diversidad. España: Comunidad Educativa.

Arbaugh, F. (2003). Study groups: Professional growth through collaboration. The Mathematics Teacher, 96(3), 188-191.

Austin, V. L. (2001). Teachers' beliefs about co-teaching. Remedial \& Special Education, 22(4), 245-255.

Balanskat, A., Blamire, R., \& Kefala, S. (2006). The ICT impact report. A review of studies of ICT impact on schools in Europe. Brussels: European Schoolnet.

Berenice, A., De Aguinaga, P., Ávila, C. (2010). El trabajo colaborativo y la inclusión social. Apertura, 2(1), 48-59.

Brito, V. (2004). El foro electrónico: Una herramienta tecnológica para facilitar el aprendizaje colaborativo. Edutec - Revista Electrónica de Tecnología Educativa, 17. Recuperado de: http://www.edutec.es/revista/index.php/ edutece/article/view/532/266

Cabezas, M., \& Casillas, S. (2009). La Web 2.0: Contexto pedagógico y utilidades didácticas. Papeles Salmantinos de Educación, 13, 247-266.

Castells, M. (2011). Autocomunicación de masas y movimientos sociales en la era de Internet. Anuari del Conflicte Social 2011, 11-19. Recuperado de: http://revistes.ub.edu/index.php/ ACS/article/view/6235

Cebrián, M. (2008). La Web 2.0 como red social de comunicación e información. Estudios sobre el mensaje periodístico, 14, 345-361.

De la Torre, A. (2006) Web educativa 2.0. Edutec - Revista Electrónica de Tecnología Educativa, 20(6). Recuperado de: http://www.edutec.es/revista/index.php/ edutec-e/article/view/515/248

Ertmer, P. A., \& Ottenbreit-Leftwich, A. T. (2010). Teacher technology change: How knowledge, confidence, beliefs and culture intersect. Journal of Research on Technology in Education, 42(3), 255-284.

García-Valcárcel, A., Hernández, A., \& Recamán, A. (2012). La metodología del aprendizaje colaborativo a través de las TIC: Una aproximación a las opiniones de profesores y alumnos. Revista Complutense de Educación, 1, 161-188. 
Hew, K. F., \& Brush, T. (2007). Integrating technology into K-12 teaching and learning: Current knowledge gaps and recommendations for future research. Educational Technology Research Development, 55(3), 227-243.

Jiménez, K. (2009). Propuesta estratégica y metodológica para la gestión en el trabajo colaborativo. Revista Educación, 33(2), 95-107.

Johnson, D. W., \& Johnson, R. T. (1987). Learning together and alone. NJ: Prentice Hall.

Johnson, D. W., Johnson, R. T., \& Smith, K. A. (1998). Active learning: Cooperation in the college classroom. Edina, MN: Interaction Book Company.

Marquès, P. (2007). La Web 2.0 y sus aplicaciones didácticas. Recuperado de: http://www.peremarques.net/web20.htm

Montero Mesa, L. (2011). El trabajo colaborativo del profesorado como oportunidad formativa. CEE Participación Educativa, 16, 69-88. Recuperado de: http://www.mecd.gob.es/revista-cee/ pdf/n16-montero-mesa.pdf

Muñoz, J. F., Quintero, J., \& Munévar, R. A. (2002). Experiencias en investigaciónacción-reflexión con educadores en proceso de formación. Revista Electrónica de Investigación Educativa, 4(1), 1-15. Recuperado de: http://redie.uabc.mx/ redie/article/viewFile/51/93

Rosario, H. (2008). La Web: Herramienta de trabajo colaborativo. "Experiencia en la Universidad de Carabobo". Pixel-Bit - Revista de Medios y Educación, 31, 131-139.

Serafí, A. (1999). El trabajo en equipo de los profesores y profesoras: Factor de calidad, necesidad, y problema. El papel de los directivos escolares. Educar, 24, 89-110.

Sigalés, C., Mominó, J. M., Meneses, J., \& Badía, A. (2008). La integración de Internet en la educación escolar española: Situación actual y perspectivas de futuro. Barcelona: UOC.

Trujillo, J. M., Cáceres, M. P., Hinojo, F. J., \& Aznar, I. (2011). Aprendizaje cooperativo en entornos virtuales. El proyecto 'Redes Educativas y Organizativas Interuniversitarias'. Educar, 47(1), 95-119.

Valverde, J., Garrido, M. C., \& Fernández, R. (2010). Enseñar y aprender con tecnologías: Un modelo teórico para las buenas prácticas educativas con TIC. Revista Electrónica Teoría de la Educación: Educación y Cultura en la Sociedad de la Información, 11(1), 203-229. Recuperado de: http://campus.usal.es/ $\sim$ revistas_trabajo/ index.php/revistatesi/article/view/5840

Williams, A., Prestage, S., \& Bedward, J. (2001). Individualism to collaboration: The significance of teacher culture to the induction of newly qualified teachers. Journal of Education for Teaching: International Research and Pedagogy, 27(3), 253-267.

\section{Legislación}

ORDEN EDU/1761/2009, de 26 de agosto, por la que se efectúa convocatoria para la concesión de la certificación en la aplicación de las Tecnologías de la Información y la Comunicación (TIC) para centros docentes públicos no universitarios de la Comunidad de Castilla y León para el curso 2009/2010 (BOCyL, ${ }^{\circ}$ 167, pp.26554-26605, martes, 1 de septiembre de 2009). URL: http://goo.gl/bMBReC Fecha de BOCyL.: martes, 1 de septiembre de 2009. 


\title{
EXPERIÊNCIAS DE TRABALHO COLABORATIVO ENTRE PROFESSORES ATRAVÉS DAS TECNOLOGIAS DE INFORMAÇÃO E COMUNICAÇÃO (TIC)
}

\section{Resumo}

Durante os anos 2011-2015, o Grupo de Pesquisa-Inovação em Tecnologia Educacional da Faculdade de Educação da Universidade de Salamanca realizou uma pesquisa, financiada pelo Ministério da Ciência e Inovação, com o objetivo de conhecer as conceções, metodologias e práticas profissionais dos professores do Ensino Primário e Secundário sobre a aprendizagem colaborativa através das Tecnologias de Informação e Comunicação, no contexto espanhol, na denominada Escola 2.0. Este artigo centra-se na análise de uma parte dos resultados: o estudo das experiências de trabalho colaborativo mediado por TIC realizadas entre professores do ensino primário e secundário. Utilizou-se uma abordagem metodológica mista (quantitativa e qualitativa) e um método não experimental e descritivo. Salientamos, como conclusão principal, que o trabalho colaborativo por meio das TIC entre os docentes é ainda muito escasso e pobre.

Palavras-chave

Inovação educacional; Escola 2.0; Trabalho colaborativo; TIC

\section{EXPERIENCES OF COLLABORATIVE WORK BETWEEN TEACHERS USING INFORMATION AND COMMUNICATION TECHNOLOGY (ICT)}

\author{
Abstract \\ During the years 2011-2015, the Research-Innovation Group in Educational \\ Technology at the Faculty of Education of the University of Salamanca in Spain \\ has conducted a research, funded by the Ministry of Science and Innovation, \\ which was focused on the concepts, methodologies and professional practices \\ of teachers in Primary and Secondary School on collaborative learning
}


through ICT's, in the context of the so-called School 2.0. This article focuses on the analysis of a single part: the study of the experiences of collaborative work using ICT between Primary and Secondary teachers. A mixed methodological approach (quantitative and qualitative) and a nonexperimental and descriptive method was used. The main conclusion indicates that the culture of collaborative work mediated by ICT among teachers is usually poor and scarce.

Keywords

Educational innovation; School 2.0; Collaborative work; ICT

Recebido em junho/2015

Aceite para publicação em fevereiro/2016

i Departamento de Didáctica, Organización y Métodos de Investigación, Facultad de Educación, Universidad de Salamanca, España.

ii Departamento de Didáctica, Organización y Métodos de Investigación, Facultad de Educación, Universidad de Salamanca, España.

iii Departamento de Didáctica, Organización y Métodos de Investigación, Facultad de Educación, Universidad de Salamanca, España.

Toda a correspondência relativa a este artigo deve ser enviada para: Marcos Cabezas González, Facultad de Educación Universidad de Salamanca, Paseo de Canalejas, 169, 37008, Salamanca, España. E-mail: mcabezasgo@usal.es 